\section{REFERENCES}

1. H. Salt, Transient conduction in two-dimensional composite slab-I. Theoretical development of temperature modes, Int. J. Heat Mass Transfer 26, 1611-1616 (1983).

2. H. Salt, Transient conduction in two-dimensional composite slab-II. Physical interpretation of temperature modes, Int. J. Heat Mass Transfer 26, 1617-1623 (1983).

3. M. D. Mikhailov, General solutions of heat equation in finite regions. Int. J. Engng Sci. 10, 577-591 (1972).
4. M. D. Mikhailov and M. N. Ozisik, Unified Analysis and Solutions of Heat and Mass Diffusion. John Wiley, New York (1984).

5. M. D. Mikhailov and N. L. Vulchanov, A computational procedure for Sturm-Liouville problems, J.comp. Phys. 50, 323-336 (1983).

6. M. D. Mikhailov, M. N. Özişik, and N. L. Vulchanov, Transient heat diffusion in one-dimensional composite media and automatic solution of the eigenvalue problem, Int. J. Heat Mass Transfer 26, 1131-1141 (1983).

\title{
On the similarity solutions to laminar natural convection boundary layers
}

\author{
Poul S. Larsen* and Vedat S. Arpaci† \\ * Department of Fluid Mechanics, Technical University of Denmark, DK-2800 Lyngby, Denmark \\ † Department of Mechanical Engineering and Applied Mechanics, University of Michigan, Ann Arbor, \\ MI 48109, U.S.A.
}

\section{INTRODUCTION}

DimENSIONAL analysis shows

$$
\Pi_{x}=\operatorname{Ra} a_{x} \operatorname{Pr} /(1+\operatorname{Pr}),
$$

in the usual nomenclature, to be the natural parameter incorporating buoyancy and the weighted contributions of both inertial and viscous effects into the correlation of heat transfer in natural convection from the vertical isothermal plate. The parameter ensures the correct limits for large and small values of the Prandtl number, where $N u_{x} / \Pi_{x}^{1 / 4}$ becomes constant. Accordingly, in terms of the similarity variable

$$
\eta=(y / x)\left(\Pi_{x} / 4\right)^{1 / 4},
$$

temperature distributions prove to be nearly similar for all fluids. The analysis suggests an appropriate expression for dimensionless wall friction, pointing to an approximate analogy between heat transfer and friction.

\section{SIMILARITY VARIABLE BY DIMENSIONAL ANALYSIS}

The reduction of the number of independent variables by one for a problem governed by partial differential equations requires the search for a similarity variable which appropriately combines two of the independent variables. Among the several methods available for this search, that of dimensional analysis may be extended by a judicious account of the physical similitude of governing equations. The extension employs the concept of two-length dimensional analysis [1]. This approach may give a similarity variable that carries an appropriate weight of several physical parameters in addition to the proper combination of two independent variables. We illustrate this observation by considering the classical problem of natural convection from the vertical isothermal plate in a Newtonian fluid for which the viscous dissipation may safely be neglected [2]. This problem was recently reviewed by Martin [3]

Let $u$ and $\theta \sim T_{w}-T_{\infty}$ denote the velocity and temperature, respectively, and $\delta \sim y$ and $l \sim y$ denote the lengths characterizing flux and flow, respectively. Equating flow to flux in the balance of thermal energy gives the characteristic velocity

$$
u \sim l a / \delta^{2},
$$

which is not externally imposed but is determined by the problem.

Balancing buoyancy against the sum of inertial and viscous forces in the balance of momentum gives

$$
\frac{f_{\mathrm{B}}}{f_{\mathrm{I}}+f_{\mathrm{V}}} \sim \frac{g \beta \theta}{u^{2} / l+v u / \delta^{2}}=\frac{g \beta \theta l / u^{2}}{1+v l /\left(u \delta^{2}\right)},
$$

or, after eliminating $u$ by equation (3),

$$
\frac{f_{\mathrm{B}}}{f_{1}+f_{\mathrm{V}}} \sim \frac{\operatorname{Pr}}{1+\operatorname{Pr}} \frac{g \beta \theta l^{3}}{a v}\left(\frac{\delta}{l}\right)^{4} \sim \frac{\operatorname{Pr}}{1+\operatorname{Pr}} \operatorname{R} a_{x}(y / x)^{4} .
$$

Equation (5) suggests the similarity variable stated by

\section{NOMENCLATURE}

a thermal diffusivity

$C$ constant

$g$ acceleration of gravity

$l$ reference length in $x$

$N u_{x}$ local Nusselt number

$\operatorname{Pr}$ Prandtl number, $v / a$

$R a_{x}$ local Rayleigh number, $g \beta\left(T_{w}-T_{\infty}\right) x^{3} /(a v)$

$T_{w}-T_{\infty}$ temperature difference between plate and free stream

$u \quad$ velocity component in $x$

$x$ coordinate from leading edge $y$ coordinate normal to plate.

Greek symbols

$\beta$ coefficient of thermal expansion

$\delta$ reference length in $y$

$\eta \quad$ similarity variable, equation (2)

$\zeta$ dimensionless streamfunction, equation (6)

$\theta$ dimensionless temperature, equation (6)

$v \quad$ kinematic viscosity

$\Pi_{x}$ dimensionless number, equation (1)

$\rho$ density

$\tau_{w} \quad$ wall shear stress. 
equation (2). In terms of this variable and

$$
\left(T-T_{\infty}\right) /\left(T_{w}-T_{\infty}\right)=\theta(\eta), \quad u=(4 a / x)\left(\Pi_{x} / 4\right)^{1 / 2} \zeta^{\prime}(\eta),
$$

the classical differential formulation [4] transforms as

$$
\begin{gathered}
\frac{\operatorname{Pr}}{1+\operatorname{Pr}} \zeta^{\prime \prime \prime}+\frac{1}{1+\operatorname{Pr}}\left(3 \zeta \zeta^{\prime \prime}-2 \zeta^{\prime 2}\right)+\theta=0, \\
\theta^{\prime \prime}+3 \zeta \theta^{\prime}=0,
\end{gathered}
$$

subject to

$$
\zeta(0)=\zeta^{\prime}(0)=\zeta^{\prime}(\infty)=0, \quad \theta(0)=1, \quad \theta(\infty)=0 .
$$

Clearly, since the Prandtl number appears explicitly in equations (7) solutions are expected to be of the form

$$
\zeta=\zeta(\eta ; P r), \quad \theta=\theta(\eta ; P r) .
$$

The definition of the local heat transfer in terms of the Nusselt number, $N u_{x}=-x \partial \theta(x, 0) / \partial y$, suggests the dimensionless heat transfer may be expressed as

$$
\frac{N u_{x}}{\left(\Pi_{x} / 4\right)^{1 / 4}}=-\theta^{\prime}(0 ; P r)
$$

Similarly, evaluating $\tau_{\mathrm{w}} / \rho=v \partial u(x, 0) / \partial y, \rho$ denoting the density and $\tau_{w}$ the wall shear; dimensionless wall friction is conveniently (as explained later) expressed as

$\frac{\tau_{w} / \rho}{\left(4 a v / x^{2}\right)\left(\Pi_{x} / 4\right)^{3 / 4}}\left(\frac{P r}{1+P r}\right)^{1 / 2}=\zeta^{\prime \prime}(0 ; P r)\left(\frac{P r}{1+P r}\right)^{1 / 2}$.

Figure 1 shows existing numerical results from refs. [4] and [5] replotted according to equations (11) and (12). Figures 2 and 3 show corresponding dimensionless distributions of temperature and velocity.

\section{DISCUSSION}

First, concerning the local heat transfer, it is well known that for $\operatorname{Pr} \rightarrow \infty, N u_{x} / R a_{x}^{1 / 4}=C_{\infty}(=0.5027)$ as inertial terms can be neglected in a creeping flow. Also, for $\operatorname{Pr} \rightarrow 0$, $N u_{x} /\left(\operatorname{Pr} R a_{x}\right)^{1 / 4}=C_{0}(=0.6004)$ is to be expected as the effect of viscosity should disappear from the solution. (Note that the same relations, with constants multiplied by $4 / 3$, are valid for the average heat transfer over length 0 to $x$ of the plate.) The similarity variable $\eta$ of equation (2), through the parameter $\Pi_{x}$, appropriately incorporates these two constant limits into the formulation of the problem. As a result the effect of the remaining explicit $\mathrm{Pr}$-dependence appearing in equation (7) is small, as seen from the solution in Fig. 1. The fact that the explicit Pr-dependence should appear in the momentum

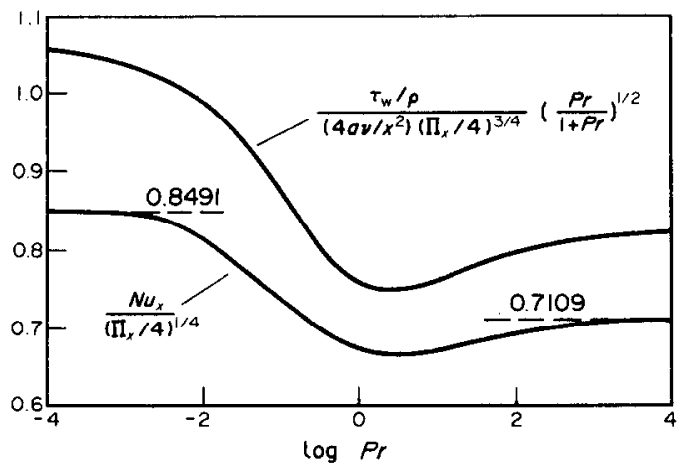

Fig. 1. Local dimensionless heat transfer and wall friction vs Prandtl number for natural convection from the vertical isothermal plate.

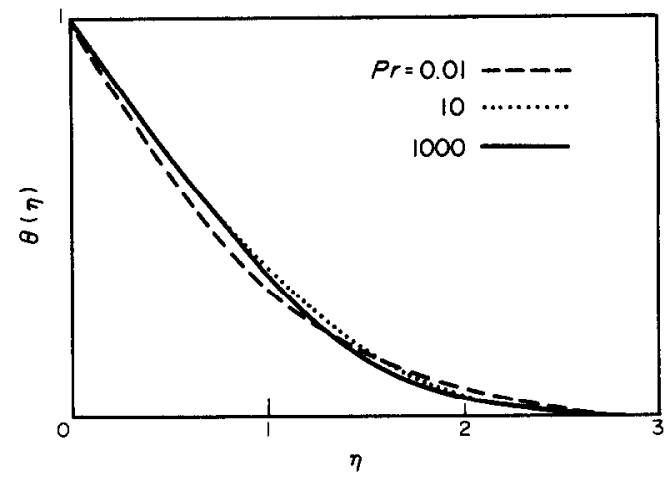

FIG. 2. Dimensionless temperature distributions for a range of Prandtl numbers.

equation to correctly reflect the physics was apparently first recognized by Le Fevre [6] who employed the present transformation (to within a constant) to obtain asymptotic solutions for $\operatorname{Pr} \rightarrow 0$ and $\operatorname{Pr} \rightarrow \infty$. Also, Sparrow et al. [7] employed similarity variables identical to the large Prandt number limit of those of the present study.

Regarding the remaining weak Pr-dependence, recall that equation (5) was derived by arbitrarily setting the ratio of viscous to inertial forces equal to $\mathrm{Pr}$, yielding the factor $\mathrm{Pr} /$ $(1+P r)$ appearing in $\Pi_{x}$. Apparently the foregoing ratio has a more complex $P r$-dependence. For example, selecting the factor to be $\operatorname{Pr} /\left(C_{1}+\operatorname{Pr}\right)$ in $\Pi_{x}$, where $C_{1}$ is a constant, would lead to equation (11) and guarantee the correct asymptotic solutions. The particular choice $C_{1}=\left(C_{\infty} / C_{0}\right)^{4}=0.4914$ makes the correlation $N u_{x} / \Pi_{x}^{1 / 4}=C_{\infty}$ valid asymptotically for $\operatorname{Pr} \rightarrow \infty$ and $\operatorname{Pr} \rightarrow 0$, where $C_{\infty}=0.5027$. However, near $P r=1$ the correlation is about $12 \%$ in error. An improved empirical fit to computer solutions for all values of $\mathrm{Pr}$ proposed by Le Fevre (see ref. [5]) amounts to employing the factor $\operatorname{Pr} /(0.4916+0.9816 \sqrt{P r}+P r)$ in $\Pi_{x}$. Clearly, any fractional power series in the denominator of this factor, truncated at the first power of $\mathrm{Pr}$, would preserve the correct asymptotic limits.

Turning next to the wall friction, which is of more theoretical interest, the case $\operatorname{Pr} \rightarrow \infty$ presents no problem. Here the contribution from inertia is altogether negligible and any explicit $P r$-dependence disappears, hence $\zeta^{\prime \prime}(0 ; P r) \rightarrow$ $\zeta^{\prime \prime}(0)$ and the RHS of equation (12) approaches a constant.

For the case $\operatorname{Pr} \rightarrow 0$, the explicit $P r$-dependence is confined to a thin layer of thickness $\eta \sim P r^{1 / 2}$ near the wall. However,

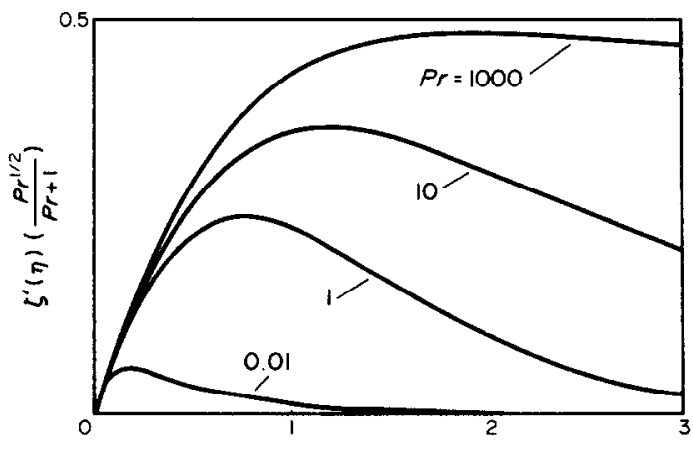

Fig. 3. Dimensionless velocity distributions for a range of Prandtl numbers. 
since the heat transfer becomes a constant the Pr-dependence does not affect the temperature distribution which is governed by the solution far from the wall. To show that the right of equation (12) approaches a constant for $\mathrm{Pr} \rightarrow 0$, consider the matched asymptotic solutions of the velocity distribution. In the usual limiting process $\operatorname{Pr}$ disappears from (7) and (8), yielding the outer solution which is independent of Pr. For the inner solution, using the fact that $\theta(\eta ; P r) \rightarrow \theta(0)$, independent of $P r$, the explicit $P r$-dependence disappears from equation (7) provided the new variable $\mathrm{Pr}^{-1 / 2} \zeta$ as function of $\mathrm{Pr}^{-1 / 2} \eta$ is employed. Consequently, $\operatorname{Pr}^{1 / 2} \zeta^{\prime \prime}(0 ; P r)$ becomes independent of $\mathrm{Pr}$ as $\mathrm{Pr} \rightarrow 0$, suggesting the expression for dimensionless friction given by equation (12).

The remaining $P r$-dependence in dimensionless friction is small, as seen from Fig. 1, and appears to be analogous to that of the heat transfer. The Pr-dependence may of course be further reduced by empirical fit to computer results in a manner similar to that discussed for the heat transfer.

The distributions of dimensionless temperature and velocity of Figs. 2 and 3 show how the present dimensionless variables nearly eliminate variations in wall gradients. The significant changes in velocity distributions with Prandtl number far from the wall appear to have a marginal effect on temperature distributions which prove to be nearly similar for all fluids.
Finally, it is noted from the ratio of equations (11) and (12) that an appropriate parameter group expressing an analogy between heat transfer and friction is, for the present problem,

$$
\frac{N u_{x} R a_{x}^{1 / 2}}{\left(\tau_{w} / \rho\right)\left(x^{2} / a v\right)}
$$

This parameter group varies less than $10 \%$ over the complete range of Prandtl numbers.

\section{REFERENCES}

1. V. S. Arpaci and P. S. Larsen, Convection Heat Transfer. Prentice-Hall, Englewood Cliffs, NJ (1984).

2. B. Gebhart, J. Fluid Mech. 14, 225-232 (1962).

3. B. W. Martin, Int. J. Heat Mass Transfer 27, 1583-1586 (1984).

4. S. Ostrach, NACA Report 1111 (1953).

5. A. J. Ede, Advances in Heat Transfer (Edited by T. F. Irvine, Jr and J.P. Hartnett), Vol. 4, pp. 1-64. Academic Press, New York (1967).

6. E. J. Le Fevre, Proc. 9th Int. Congress Applied Mechanics, Brussels, Vol. 4, pp. 168-174 (1956).

7. E. M. Sparrow, F. K. Tsou and E. F. Kurtz, Phys. Fluids 8, 1559 (1965).

\title{
Mixed convection from a horizontal line source of heat
}

\author{
Ramesh Krishnamurthy and Benjamin GebHaRT \\ Department of Mechanical Engineering and Applied Mechanics, University of Pennsylvania, Philadelphia, \\ PA 19104, U.S.A.
}

(Received 30 October 1984 and in final form 3 September 1985)

\section{INTRODUCTION}

THE MECHANISMS of mixed convection from a horizontal line source of heat have come under active investigation relatively recently. The earliest study by Wood [1], followed by that of Wesseling [2], considered mixed convection from weakly buoyant plumes. Afzal [3] presented a complete analysis describing the entire flow regime, ranging from weakly to strongly buoyant plumes. It is well known that in a free convection plume from a line source of heat, the centerline velocity increases continuously as $x^{1 / 5}, x$ being the distance downstream of the source. Thus, even in the presence of a nonzero free-stream velocity, it appears that, at sufficiently large $x$, buoyancy effects would dominate the transport mechanism. This corresponds to a strongly buoyant plume. Such is the flow studied here, with the buoyancy force and the free-stream flow being in the same direction.

Afzal [3] considered the mixed convection from a line source in terms of two coordinate expansions, a direct coordinate expansion and an inverse, valid for small and large streamwise distance from the source, respectively. The direct coordinate expansion was taken in terms of a variable $\xi, \xi$ being proportional to $x^{1 / 2}$. The inverse coordinate expansion was constructed in terms of $\xi^{-2 / 5}$. The solution for the first 11 and the first seven terms, in the direct and inverse coordinate expansions, respectively, are presented. However, these expansions were constructed entirely on the basis of the classical boundary-layer solution. The non-boundary-layer effects, for example, the effect of the flow in the ambient, resulting from the entrainment into the boundary layer, was not considered. Under some circumstances these non- boundary-layer effects also contribute significantly. It then becomes necessary to simultaneously assess both the effects of the presence of external free-stream velocity and of the nonboundary-layer effects. The first such simultaneous and consistent assessment in the analysis of mixed convection flows was that by Carey and Gebhart [4].

The method of matched asymptotic expansions is used here to obtain a solution valid at a large downstream distance from the source. Two perturbation parameters, $\varepsilon_{\mathrm{H}}$ and $\varepsilon_{\mathrm{M}}$, characterize the non-boundary-layer and the non-zero freestream velocity effects, respectively. These effects are considered simultaneously, as perturbations of the associated natural convection plume flow. It is shown that corrections due to higher-order effects enter into the expansion before the fifth term of the inverse coordinate expansion considered by Afzal [3], for the same flow. Results of the numerical computations are presented for $\operatorname{Pr}=0.7$.

\section{ANALYSIS}

The mixed convection flow arising from an infinitely long horizontal line source of heat is considered as a twodimensional steady flow. With usual Boussinesq approximations, neglecting the viscous dissipation and pressure terms in the energy equation, the full two-dimensional governing equations take the form

$\psi_{y} \frac{\partial}{\partial x}\left(\psi_{x x}+\psi_{y y}\right)-\psi_{x} \frac{\partial}{\partial x}\left(\psi_{x x}+\psi_{y y}\right)-\nu \nabla^{4} \psi-g \beta \frac{\partial t}{\partial y}=0$ 